\title{
A GPU-Based Genetic Algorithm for the P-Median Problem
}

\author{
Bader F. AlBdaiwi and Hosam M.F. AboElFotoh \\ Computer Science Department, Kuwait University, Kuwait
}

\{bdaiwi, hosam\}@cs.ku.edu.kw

June 17, 2021

\begin{abstract}
The p-median problem is a well-known NP-hard problem. Many heuristics have been proposed in the literature for this problem. In this paper, we exploit a GPGPU parallel computing platform to present a new genetic algorithm implemented in Cuda and based on a Pseudo Boolean formulation of the p-median problem. We have tested the effectiveness of our algorithm using a Tesla K40 (2880 Cuda cores) on 290 different benchmark instances obtained from OR-Library, discrete location problems benchmark library, and benchmarks introduced in recent publications. The algorithm succeeded in finding optimal solutions for all instances except for two OR-library instances, namely pmed30 and pmed40, where better than $99.9 \%$ approximations were obtained.
\end{abstract}

Keywords P-Median Problem; NP-Hard; GPGPU; Cuda; Pseudo Boolean Formulation; Genetic Algorithms; Heuristics.

\section{Introduction}

The P-Median Problem (PMP) is formally defined as follows. Given a set $C=$ $\{1, \ldots, n\}$ of $n$ clients, a set $F=\{1, \ldots, m\}$ of $m$ facilities, an integer $p<m$, and the distance $d_{i j}$ between client $i, 1 \leq i \leq n$, and facility $j, 1 \leq j \leq m$. Let $y_{i j} \in\{0,1\}$ be a decision variable such that $y_{i j}=1$ if and only if client $i$ is serviced by facility $j$, and let $x_{j} \in\{0,1\}$ be a decision variable such that $x_{j}=1$ if and only if facility $j$ is open for service. The PMP objective is to minimize the total distance 1

$$
f_{C}(x, y)=\sum_{i \in C} \sum_{j \in F} x_{j} y_{i j} d_{i j}
$$

\footnotetext{
${ }^{1}$ We shall use distance and cost interchangeably
} 
subject to

$$
\begin{gathered}
\sum_{j \in F} y_{i j}=1 \quad \forall i \in C, \\
\sum_{j \in F} x_{j}=p .
\end{gathered}
$$

The objective function (1) minimizes the total distance between clients and the corresponding service facilities. Constraint (2) states that each client is serviced by exactly one facility. Constraint (3) states that the number of open facilities is exactly $p$. Let the set of open facilities be $O=\left\{o_{1}, \ldots, o_{p}\right\}$. Naturally, if client $i$ is serviced by facility $j,\left(y_{i j}=1\right)$, then: 1) $j \in O$ (is open), and 2) $d_{i j}$ is a minimum over $\left\{d_{i o_{1}} \ldots d_{i o_{p}}\right\}$. An instance of the PMP is described by an $n \times m$ distance matrix $C=\left[d_{i j}\right]$ and a positive integer $p<m$. Note that we assume the elements of $C$ are non-negative.

The PMP has a wide range of applications. It has been extensively researched in the literature. It has many applications in logistics [7][20] and location science [25] 34. It also has applications in finance and market analysis [15]. Unfortunately, it is NP-hard and hence difficult to solve it for optimality [23]. Comprehensive surveys on solving methods for the PMP and its variations can be referred to in 11, 14, 30, 33.

In this paper, we exploit a GPGPU parallel computing platform to present a new genetic algorithm implemented in Cuda $\mathrm{C}$ version 7.5 (Compute Unified Device Architecture) and based on a Pseudo Boolean formulation of the PMP.

The rest of the paper is organized as follows. Section 2 introduces preliminaries and related literature review on the pseudo boolean formulation of the PMP, GPGPU and Cuda, and genetic algorithms. Section 3 presents the new algorithm. Section 4 highlights some implementation details. The algorithm time complexity is analyzed in Section 5. Section 6 presents the experimentation results, and Section 7 concludes the paper.

\section{Preliminaries}

\subsection{A Pseudo Boolean Formulation of the PMP}

The pseudo Boolean formulation of the PMP appeared in [18]. It is obtained as follows. For each client $i$, let $\prod^{i}=\left(\pi_{i 1}, \ldots, \pi_{i m}\right)$ be an ordering of $1, \ldots, m$ such that $d_{i \pi_{i k}} \leq d_{i \pi_{i l}}$ if $k<l$ for all $k, l \in\{1, \ldots, m\}$, and let $\triangle^{i}=\left(\delta_{i 1}, \ldots, \delta_{i m}\right)$, where $\delta_{i 1}=d_{i \pi_{i 1}}$, and $\delta_{i r}=d_{i \pi_{i r}}-d_{i \pi_{i(r-1)}}$, for $r=2, \ldots, m$. Therefore, the distance between client $i, i \in\{1, \ldots n\}$, and the facility serving it can be expressed using the following pseudo Boolean polynomial

$$
d_{i}=\delta_{i 1}+\sum_{k=2}^{m} \delta_{i k} \prod_{r=1}^{k-1} \bar{x}_{\pi_{i r}} .
$$


Thus, the PMP can be reformulated as: Given $\triangle=\left[\delta_{i j}\right], \prod=\left[\pi_{i j}\right]$, and $p$, find an assignment in $\{0,1\}$ to $x_{i}, i \in\{1, \ldots, n\}$, such that

$$
\sum_{j \in F} x_{j}=p
$$

and

$$
B_{C}(z)=\sum_{i=1}^{n}\left(\delta_{i 1}+\sum_{k=2}^{m} \delta_{i k} \prod_{r=1}^{k-1} z_{\pi_{i r}}\right)
$$

is minimized. The Boolean variable $z_{j}=\bar{x}_{j}$ is 1 iff $x_{j}=0$, denoting a closed facility. Note that the $k^{t h}$ term in Equation (4) contains $\prod_{r=1}^{k-1} \bar{x}_{\pi_{i r}}$. Therefore, this term must be zero $\forall k>m-p+1$ since at least one facility (say $f$ ) is open in any $m-p+1$ locations resulting in $\bar{x}_{f}=0$. Thus, $\prod$ and $\triangle$ can be reduced to $\Pi^{\prime}$ and $\triangle^{\prime}$ by omitting the last $p-1$ columns.

The objective function based on $\Pi^{\prime}$ and $\triangle^{\prime}$ is known as Hammer-Bersnev polynomial $(H B P)[5]$. It can be further reduced through monomial reduction. Interested readers could refer to [4 for details. Example 1 illustrates the PMP pseudo boolean formulation.

Example 1 : Consider a PMP instance with $n=5, m=4, p=2$ and

$$
C=\left[\begin{array}{cccc}
7 & 10 & 16 & 11 \\
15 & 17 & 7 & 7 \\
10 & 4 & 6 & 6 \\
7 & 11 & 18 & 12 \\
10 & 22 & 14 & 8
\end{array}\right]
$$

An ordering matrix $\prod$ and the corresponding matrix $\triangle$ are given by

$$
\Pi=\left[\begin{array}{llll}
1 & 2 & 4 & 3 \\
3 & 4 & 1 & 2 \\
2 & 3 & 4 & 1 \\
1 & 2 & 4 & 3 \\
4 & 1 & 3 & 2
\end{array}\right], \Delta=\left[\begin{array}{llll}
7 & 3 & 1 & 5 \\
7 & 0 & 8 & 2 \\
4 & 2 & 0 & 4 \\
7 & 4 & 1 & 6 \\
8 & 2 & 4 & 8
\end{array}\right]
$$

Omitting the last $(p-1=1)$ column corresponding to zero terms in Equation (44) results in:

$$
\Pi^{\prime}=\left[\begin{array}{lll}
1 & 2 & 4 \\
3 & 4 & 1 \\
2 & 3 & 4 \\
1 & 2 & 4 \\
4 & 1 & 3
\end{array}\right], \Delta^{\prime}=\left[\begin{array}{lll}
7 & 3 & 1 \\
7 & 0 & 8 \\
4 & 2 & 0 \\
7 & 4 & 1 \\
8 & 2 & 4
\end{array}\right]
$$


The corresponding HBP representing total distance (cost) is

$$
\begin{gathered}
B_{C}(z)=\begin{array}{c}
{\left[7+3 z_{1}+1 z_{1} z_{2}\right]+\left[7+0 z_{3}+8 z_{3} z_{4}\right]+\left[4+2 z_{2}+0 z_{2} z_{3}\right]+} \\
{\left[7+4 z_{1}+1 z_{1} z_{2}\right]+\left[8+2 z_{4}+4 z_{1} z_{4}\right] .}
\end{array} \\
B_{C}(z) \text { has } n \times(m-p+1)=15 \text { entries, and the reduced polynomial is } \\
B_{C}(z)=33+7 z_{1}+2 z_{2}+2 z_{4}+2 z_{1} z_{2}+8 z_{3} z_{4}+4 z_{1} z_{4} .
\end{gathered}
$$

\subsection{GPGPU and Cuda}

Similar to many NP-hard problems, many heuristics have been developed for the PMP. Some of these heuristics tried to exploit parallel computing platforms to reach a near-optimal solution in a reasonably short time [11. A few years ago, Nvidia has introduced Cuda (Compute Unified Device Architecture) that provides an application interface (API) for general purpose computing [?]. Hence, GPGPU refers to General Purpose computing on Graphics Processing Units. Nvidia graphics cards (as well as all graphics cards) are designed to do similar computations on large numbers of pixels. Therefore, they contain hundreds of processing elements (cores), although not as powerful as CPU cores, that execute thousands of similar threads (grouped in blocks) in parallel. Unlike CPU's threads, the context switching between blocks of threads requires a minimal overhead. There are not many GPU-based solutions for the PMP so far. Lim and Ma introduced GPU implementations for solving the PMP using the vertex substitution and Llod algorithms in [26] and [27]. Cuda $\mathrm{C}$ can be used for developing applications on GPGPU. In our implementation, we used Cuda C version 7.5 .

A Cuda C program consists of two types of code: Host code and Device code. The Host code refers to that executed by the CPU and the device code refers to that executed by the GPU card. The Host code launches a kernel that is executed by the device. A kernel launch specifies the number of threads to be executed in parallel. These threads are grouped in blocks. Blocks in their turns are organized in a grid. All threads execute the same code but on multiple data, which Nvidia calls a Single Instruction Multiple Thread (SIMT) architecture. To support multidimensional data modeling and processing, Cuda enables defining grids and blocks to be single, double, or triple dimensions. For example, the following Cuda code declares two triple dimensional arrays using the dim3 data type. Then, it invokes KernelX with a $3 \times 4 \times 6$ grid each element of which is a $2 \times 4 \times 4$ block.

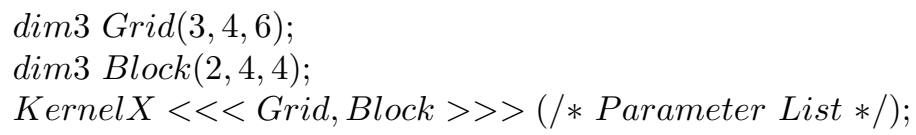

The total number of blocks in KernelX is $(3 \times 4 \times 6=72)$, and each block has $(2 \times 4 \times 4=32)$ threads. Thus, the total number of threads in KernelX is $(72 \times 32=2,304)$. To differentiate among threads, Cuda defines two built-in 
variables for block and thread indexing, namely blockIdx and threadIdx. Each of these variables is 3-dimensional. For instance, threadIdx has three components threadIdx.x, threadIdx.y, and threadIdx.z. Cuda also defines two other built-in 3dimensional variables for the grid and block dimensions, gridDim and blockDim. They are automatically initialized at a kernel launch. In the above example, KernelX sets gridDim. $x=3$, gridDim.y $=4$, and gridDim. $z=6$. It also sets blockDim. $x=2$, blockDim. $y=4$, and blockDim. $z=4$.

Linearized unique block identifier, $B I D$, and linearized unique global thread identifier, TID, can be derived from gridDim, blockDim, blockIdx, and threadIdx as follows:

$$
\begin{aligned}
& B I D=\text { blockIdx } . x+\text { blockIdx } . y * \operatorname{gridDim} . x+ \\
& \text { gridDim. } * \text { gridDim.y } * \text { blockIdx.z; } \\
& T I D=B I D *(\text { blockDim. } x * \text { blockDim.y } * \text { blockDim.z })+ \\
& \text { (threadIdx.z*(blockDim. } * \text { blockDim.y }))+ \\
& \text { (threadIdx.y*blockDim.x) + threadIdx.x; }
\end{aligned}
$$

Threads may access data in parallel from different memory locations. Usually, the data accessed by each thread is determined by its index (within the block) or its global identifier. In Cuda, the device code can access only the device memory. Therefore, the host code has to initialize the device memory through Cuda calls that allocate device memory (cudaMalloc) and copy (cudaMemcpy) data between host RAM and device RAM. The device memory has three basic types:

Global can be accessed by all threads from all blocks.

Shared can be accessed by all threads in the block. Each block has a limited amount of shared (on-chip) memory.

Private can be accessed only by the thread itself. Each thread is allocated a limited number of registers.

The global memory is too slow compared to the shared and private memories. Therefore, the shared and private memory have to be utilized to the maximum extent.

\section{$2.3 \quad$ Genetic Algorithms}

One of the most suitable heuristics framework (Meta Heuristics) that exploits the availability of many cores performing the same instruction thread on multiple data is Genetic Algorithms (GA) [29]. Recently, efficient GPU-based GA are being proposed for solving hard problems. For example, Kang et. al. have introduced such a solution for the Traveller Salesman Problem (TSP) in [22. We have not encountered any GPU-based GA for the PMP; even though, GA are recognized as one of the most effective evolutionary technique for solving optimization problems. 
In GA, a large number (Population) of Chromosomes are generated and operated upon using similar operations like mutations, crossover, migration and fitness test. A chromosome is a finite sequence of genes commonly represented by a binary string or a set of integers. A crossover operation involves two parent chromosomes exchanging genes to produce offsprings, while a mutation involves only a single chromosome that mutates into a new one. Usually, each chromosome represents a candidate solution to the optimization problem, and the fitness of the chromosome represents the solution quality or the objective function value.

There exists a number of GA for solving the PMP in the literature, examples are [6] 10] 21]. Combining GA with local search method results in hybrid GA that could speed up the reach for global optima [13. Hybrid GA for solving the PMP appear in 32 35. Hybrid GA based on the variable neighborhood search have been recently published in [12] 37]. Different hybrid GA for the PMP using solution archiving, greedy strategy, and fine-grained tournament selection are presented in 9] 24] and [36, respectively.

\section{A New Genetic Algorithm for the PMP Based on GPU and Pseudo Boolean Formulation}

The algorithm is quite simple. The host randomly generates an initial population of chromosomes (candidate solutions) and passes them to a device kernel for fitness evaluations and enhancements. This basically iterates with migrating the best fit chromosomes from the current population to the next. The algorithm terminates at reaching an iteration limit or over saturating the solution enhancement.

Parallelism manifests in our algorithm in two folds. First, the host generates the next population in parallel with the device processing of the current population fitness evaluations and enhancements. Second, the kernel threads run in parallel, and each of which is assigned a chromosome for fitness evaluation and enhancement. The fitness evaluation is based on $\Pi^{\prime}$ and $\triangle^{\prime}$ whose matrix structure harnessed the PMP data parallelism potentials as explained in 4.2, A chromosome enhancement is based on crossover and mutation operations. The details of these operations are explained in Subsections 4.3 and 4.4 .

The following two subsections outline the algorithm host and device codes.

\subsection{Host Code}

$n$ : Number of Clients, $m$ : Number of Facility Locations, $p$ : Number of Open Facilities, $C: n \times m$ Distance/Cost Matrix

$N B$ : Number of GPU Blocks

$N T$ : Number of Threads per Block 
EvolveLimit: Limit on Number of Calls to Evolve Kernel

$S$ : Saturation Limit

\section{Steps:}

1. Read input $n, m, p, C, N B, N T$, KernelsLimit.

2. Call kernel (Init $<<<N B, N T>>>$ ) to differently seed curand in each thread.

3. Compute $\prod$ and $\triangle$ matrices each of size $(n \times m)$, and reduce them to $\prod^{\prime}$ and $\triangle^{\prime}$ each of size $(n \times m-p+1)$.

4. Allocate device memory for $\prod^{\prime}$ and $\triangle^{\prime}$ using cudaMalloc.

5. Copy $\Pi^{\prime}$ and $\triangle^{\prime}$ to device memory using cudaMemcpy.

6. Initialize a counter for the Number of Evolve kernal calls: NKernels $=0$.

7. Generate candidate solutions as a random population of $N B \times N T$ chromosomes.

8. Wait for Init kernel to finish (cudaDeviceSynchronize).

9. Copy current population to device memory (cudaMemcpy).

10. Call kernel (Evolve $<<<N B, N T>>>$ ).

11. Generate a new random population of $N B \times N T$ chromosomes for next Evolve kernel call.

12. Wait for Evolve kernel to finish (cudaDeviceSynchronize).

13. Copy most fit chromosome (best solution) of each block to the Host memory and find their most fit.

14. Increment NKernels.

15. If (the most fit chromosome has not been changed by the last $S$ Evolve kernal call) or (NKernels $>=$ EvolveLimit), report the most fit chromosome as the best solution and stop;

16. Else Migrate $N B$ best chromosomes to next population and Go to Step 9.

17. END (Host Code). 


\subsection{Device Code (Executed in Parallel by Each Thread)}

\subsubsection{Init Kernel}

\section{Steps:}

1. Call curand_init(0, TID, O, Estate), (Thread Global Identifier) is passed as a sequence number [3]. This insures each thread to have a different random sequence when calling curand.

2. END (Init Kernel).

\subsubsection{Evolve Kernel}

\section{Global Memory:}

- Array B_MinCost[NB]: Minimum cost (Highest fitness) found by each block.

- Array Best[NB]: Chromosome with best fitness value for each block.

\section{Shared Memory:}

- Array MinCost[NT] : Minimum cost found by each thread in the block, initially set to MinCost[0].

- txMin[NT]: used to find the Index of the thread with best fitness value in the block, initially set to threadIdx.x.

\section{Steps:}

1. Evaluate the fitness of the thread chromosome $\mathbb{C}$ using $\prod^{\prime}$ and $\triangle^{\prime}$ matrices.

2. Initialize relative thread index and relative block size: $r t x=$ threadIdx.x; rb_size $=\mathrm{NT}$.

3. Crossover Cycle:

For $($ CStride $=\mathrm{NT} / 2 ;$ CStride $>0 ;$ CStride $=$ CStride $/ 2)$

(a) Generate a random crossover point r1 (using curand).

(b) If $\left(\mathrm{rtx}>=\mathrm{rb} \_\right.$size $\left./ 2\right)$, Stride $=$-CStride else Stride $=$ CStride.

(c) Form the parent couples by finding a unique couple for each thread: Couple $=$ TID + Stride;

(d) Make cross-over between $\mathbb{C}$ and Couple at r1 and form offspring $\mathcal{F}$.

(e) If $(\operatorname{Fitness}(\mathcal{F})<\operatorname{Fitness}(\mathbb{C}))$, replace $\mathbb{C}$ by $\mathcal{F}$.

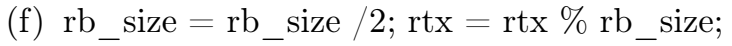

4. Synchronize each block threads using syncthreads(). 
5. Mutation Cycle:

For $(\mathrm{i}=\lg (\mathrm{NT})$, Enhanced $=$ False; $(\mathrm{i}>0)$ and $($ Not Enhanced $) ; \mathrm{i}=\mathrm{i}-1)$

(a) Randomly decide the mutation parameters as per the details explained in 4.4

(b) Mutate $\mathbb{C}$ to offspring $\mathcal{F}$.

(c) If $\operatorname{Fitness}(\mathcal{F})<\operatorname{Fitness}(\mathbb{C})$,

i. Replace $\mathbb{C}$ by $\mathcal{F}$.

ii. Enhanced $=$ True.

6. Synchronize each block threads using syncthreads().

7. Find best fitness in the block:

For $($ Stride $=\mathrm{NT} / 2 ;$ Stride $>0 ;$ Stride $=$ Stride $/ 2)$

(a) $t x=$ threadIdx.x.

(b) If ( $\mathrm{tx}<$ Stride and $($ MinCost $[\mathrm{tx}+$ Stride $]<\operatorname{MinCost}[\mathrm{tx}]))$

i. $\operatorname{MinCost}[\mathrm{tx}]=\operatorname{MinCost}[\mathrm{tx}+$ Stride $]$.

ii. $\operatorname{txMin}[\mathrm{tx}]=\mathrm{txMin}[\mathrm{tx}+$ Stride $]$.

8. If (threadIdx $=0$ ), Store each block best fitness cost and its corresponding chromosome in B_MinCost[blockIdx.x] and Best[blockIdx.x], respectively.

9. End (Evolve Kernel).

\section{Implementation Details}

\subsection{Chromosome Representation and Generation}

A chromosome is represented as a vector $\mathbb{C}$ of $m$ bits $\mathbb{C}_{0}: \mathbb{C}_{m-1}$, where true denotes an open facility and false denotes a closed one. Our algorithm generates chromosomes by random selection from a lexicographical order of a combinatorial sequence [17]. For each chromosome, it first generates a non-negative integer $i<\left(\begin{array}{c}m \\ p\end{array}\right)$ using a 64-bit random function. Next, it generates the $i^{\text {th }}$ Lexicographic combination of $\left(\begin{array}{c}m \\ p\end{array}\right)$ using the efficient method presented in 28. As a result, the generated chromosome will have exactly $p$ true bits each of which corresponds to a selected element in the $i^{\text {th }}$ Lexicographic combination of $\left(\begin{array}{c}m \\ p\end{array}\right)$. This method reduces the random function calls to one call per chromosome generation. Hence, it could maintain better quality random number generation as it limits the probability of exhaustively consuming the pseudo random sequence generated by the utilized random function. It has positively impacted the quality of the solutions generated by our algorithm. Interestingly, we are not aware of its existence in the literature. 


\subsection{Fitness Function}

The Fitness function is a performance bottleneck. Each thread calls it several times. It is called to evaluate a thread assigned chromosome. It is also called in each crossover iteration to evaluate the offsprings. Furthermore, it is called to evaluate the mutation offsprings. Therefore, we designed this function to be as efficient as possible. In general, we harnessed the data parallelism in the PMP by using the pseudo boolean formulation and tailored it to be GPU suitable. We designed the fitness function to use $\Pi^{\prime}$ and $\triangle^{\prime}$ rather than HBP. This enabled higher degree of data parallelism and restricted the required operations to be simple integer additions. We also took advantage of memory caching when accessing the $\Pi^{\prime}$ and $\triangle^{\prime}$ simply because all threads in all blocks use exactly the same $\prod^{\prime}$ and $\Delta^{\prime}$ in read only mode.

The function algorithm is straightforward. For an input chromosome $\mathbb{C}$, it scans the entry of each client in $\triangle^{\prime}$ as per the order of $\prod^{\prime}$ and accumulates the corresponding increments till an open facility is found $\left(\mathbb{C}_{\mathbb{S}}=\right.$ true $)$. The total accumulations of all clients represents the fitness of $\mathbb{C}$.

function Fitness ( $\mathbb{C}:$ Chromosome )

1. fitness $=0$;

2. for each client $i, 1 \leq i \leq \mathrm{n}$ :

- $S=0$;

- repeat: $S++$; fitness $=$ fitness $+\delta_{i \pi_{i s}}$ until $\mathbb{C}_{\mathbb{S}}$

\section{End (Fitness).}

The Fitness function time order is $O(n(m-p))$ since each of $\prod^{\prime}$ and $\triangle^{\prime}$ is of size $n \times(m-p+1)$. In average, there will be one open facility in each $\frac{m-p-1}{p} \approx \frac{m}{p}$ locations assuming the $p$ open facilities are normally distributed. Therefore, the Fitness function expected average time is $O\left(\frac{n m}{p}\right)$. Based on this, we decided to compute each chromosome fitness by a single thread accumulating the increments of all clients. This results in a better device core utilization and higher scalability as explained in Section [6. Consequently, we designed the Evolve kernel grid and blocks to be of single dimensions.

\subsection{Crossover Operation}

Each thread determines its unique couple as in Step 3 of the Evolve kernel. One of the couple threads generates two random integers and shares them with its couple. The first integer $r 1,0 \leq r 1<m$, determines the crossover starting index. The second integer $r 2=2 i, 0<i \leq\left\lfloor\frac{p}{2}\right\rfloor$, determines the number of genes to be exchanged. The exchanges count and occur only between unequal corresponding genes. In order to keep exactly $p$ true genes in the offspring, exactly $i$ genes are exchanged from 0 to 1 , and the other remaining $i$ genes are 


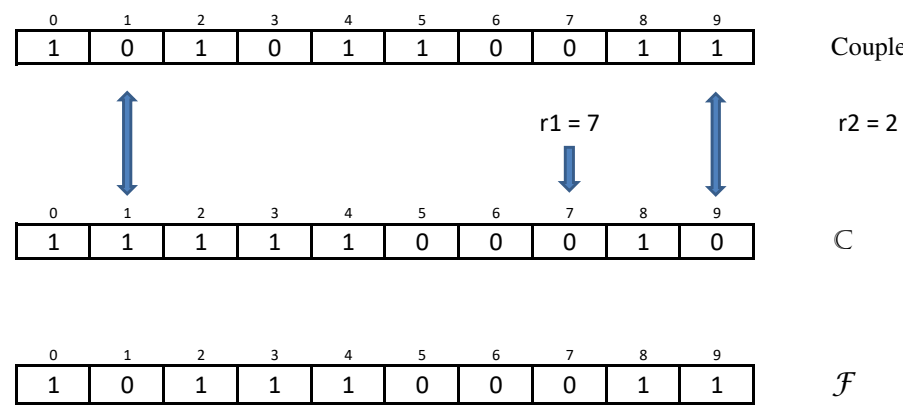

Figure 1: A crossover operation between $\mathbb{C}$ and Couple to offspring $\mathcal{F}, m=10$, $p=6, r 1=7$, and $r 2=2$.

\begin{tabular}{|l|l|l|l|l|l|l|l|l|l|}
\hline 0 & 1 & 2 & 3 & 4 & 5 & 6 & 7 & 8 & 9 \\
\hline 1 & 0 & 1 & 0 & 1 & 1 & 0 & 0 & 1 & 0 \\
\hline 0 & 1 & 2 & 3 & 4 & 5 & 6 & 7 & 8 & 9 \\
\hline
\end{tabular}
\begin{tabular}{|l|l|l|l|l|l|l|l|l|}
\hline 0 & 1 & 0 & 1 & 0 & 1 & 1 & 0 \\
\hline
\end{tabular}

Figure 2: Three positions right circular shift mutation of $\mathbb{C}$ to offspring $\mathcal{F}$.

exchanged from 1 to 0 . If the end of the chromosome is reached before having the right number of exchanges, the search continues from the beginning. If the the number of exchanges cannot reach $r 2$, the operation fails and no offspring is produced. Figure 1 shows a crossover operation example.

\subsection{Mutation Operation}

The mutation operation is based on gene/bit shifting. We use two types of shifts: circular shift and block shift. In circular shift the number of genes to be shifted and the shift direction are randomly decided. Then, the genes are rotated accordingly. Figure 2 illustrates a three positions right circular shift mutation of chromosome $\mathbb{C}$ to offspring $\mathcal{F}$.

A block shift is a circular shift on a randomly selected subsequence of the chromosome to be mutated. The number of positions to be shifted, the shift direction, and the subsequence indexes are randomly decided. Then, the subsequence genes are rotated accordingly. Figure 3 shows one position left block shift on subsequence 3 to 6 of chromosome $\mathbb{C}$ to offspring $\mathcal{F}$.

\subsection{Migration Operation}

Cuda does not support thread synchronizations across different blocks. It only supports synchronizations of threads within the same block. Therefore, we had to implement the migration operation in the Host code. The migration takes place by selecting the best fit chromosome computed by each block in the last Evolve kernel and adding it to the newly generated population for the next 


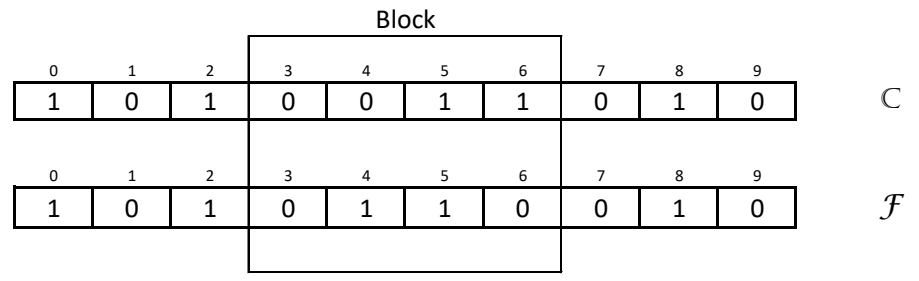

Figure 3: One position left block shift mutation of $\mathbb{C}$ to offspring $\mathcal{F}$.

kernel launch. Different variations of the migration operation can be applied. For example, the best of each block can be migrated to the same block in the next generation, or a team of all the bests can be migrated to a single block.

\section{Time Complexity}

The Fitness function time complexity as indicated in Subsection 4.2 is $O(n(m-$ $p))=O(n m)$. Hence, each of the crossover and mutation cycles in the Evolve kernel is $O(n m l g(N T))$. Therefore, the Evolve kernel time complexity is

$$
T_{E}=O(n m \lg (N T)) .
$$

The Init kernel is $O(1)$ since each thread would execute a constant number of operations.

The complexity of generating one chromosome (candidate solution) in the host is $O(m p)$. Consequently, the time complexity of the first eight steps in the Host Code is

$$
T_{S}=O(n m)+O(N B N T m p) .
$$

The remaining steps are iterative and the time complexity of a single iteration of these steps is

$$
T_{H}=O(N B N T m p)+W,
$$

where $W$ is the waiting time for the Evolve kernel to finish (Step 12). Since $T_{H}$ and $T_{E}$ run in parallel, the algorithm total complexity is

$$
T_{S}+O\left(\text { EvolveLimit } \times \max \left(T_{H}, T_{E}\right)\right) .
$$

For maximum utilization of both Host and GPU device, $W$ must be 0 and $T_{H}=T_{E}$. This can be achieved by proper selection of NB and NT within the GPU device limits. Further synchronization between $T_{H}$ and $T_{E}$ could be achieved by increasing the crossover and/or mutation iterations to a number decided by an input parameter. 


\section{Experimentation Results}

The objective of the experimentation was to test the effectiveness of our algorithm rather than to optimize the implementation to the best possible performance. We have tested the algorithm on all the benchmark instances we had access to. In total, we have tested 290 diversified instances collected as follows: 40 instances from the OR-Library [8], 40 instances of the so-called complex instances introduced in Table 2.6 of [16, and 210 instances from the discrete location problems benchmark library [2]. All our experimentation were executed on a Tesla K40 (2880 Cuda cores) hosted by HP Z820 workstation equipped with: $2 \times$ Intel Xeon processors 12 cores each, 16 GB RAM, and $2 \times 512 \mathrm{~GB}$ solid state drives. The specifications of these equipment could be referred to in [19] 31.

The algorithm succeeded in obtaining optimal solutions for all the 290 instances except two, namely OR-Library pmed30 and pmed40 where a better than $99.9 \%$ approximation was obtained for each. The obtained results are listed in Tables 1 to 9 . By examining these results, we can draw the following notes and observations:

1. Our algorithm succeeded in obtaining optimal solutions for all what so called "complex instances" as shown in Table 9. Goldengorin et. al. introduced these forty instances where optimal solutions for thirty of which could not be obtained by linear programming using Elloumi formulation or pseudo boolean formulation and data reductions [16].

2. Our algorithm critically relies on randomization in initializing potential solutions and enhancing them. Thus, there is no guarantee to obtain the same results in each run of the algorithm on a given benchmark instance. Except for OR-Library pmed30 and pmed40, nevertheless, our implementation has shown excellent consistency in obtaining optimal solutions over multiple runs on each tested benchmark instance, but possibly with different timings and/or kernel counters. The measurements listed in Tables 1 to 9 are the medians obtained from different runs. Furthermore, these measurements are for the kernels in which the optimal solutions were achieved rather than for the kernels at which the program terminated with the exceptions of pmed30 and pmed40 as no optimal solutions were achieved.

3. The chromosomes generation method as explained in Subsection 4.1 tremendously enhances the candidate solutions' qualities. Moreover, the independence of the random functions used in the Host Code and in the Evolve kernel contributes to this enhancement as it dedicates the host random function for generating candidate solutions.

4. As indicated in Subsection 4.2 the Fitness function is a performance bottleneck. In average, there is one open facility in any $\frac{m-p-1}{p} \approx \frac{m}{p}$ locations assuming the open facilities are normally distributed. We could have sped up this function execution by using $n$ threads each of which accumulates 
the increments of one client ( $n$ threads scenario) rather than using a single thread to accumulate the increments of all clients (single thread scenario). In the $n$ threads scenario, however, the Fitness function execution time is determined by the last finishing thread, $\left(t_{l}\right)$, whose execution time in average will be worse than $\frac{m}{p}$ and could be $\Omega(m)$. Each of the other $n-1$ threads will be idle from its finishing time till the finishing time of $t_{l}$. This would result in underutilized device cores, and would hinder the performance scalability as $n$ and $m$ increase. The single thread scenario requires more time to evaluate the fitness of a single chromosome, but with no thread idle time. In this scenario, the Fitness function average time is $O\left(\frac{n m}{p}\right)$. As $p$ scales to $\theta(m)$, the average time could drop to $O(n)$. This explains the total time drop when scaling $p$ and fixing $n$ and $m$ in our experimentation of the Pmed and the Complex benchmarks, refer to Tables 1 and 9. Evaluating the fitness of $n$ chromosomes in the $n$ threads scenario requires $n^{2}$ threads and $O(m)$ average time. The same requires $n$ threads and $O(n)$ average time with proper scaling of $p$. As the number of threads exceeds the number of available cores, thread queuing overhead and waiting times will accumulate. Obviously, the $n$ threads scenario requires more threads as $n$ scales. Thus, it is more vulnerable to these overheads and waiting times.

5. The time needed to generate a population is proportional to its size $=$ $N B \times N T$. We noticed that increasing the population size improves the chances of obtaining an optimal solution, refer to Table 6 as an example. However, determining the population size has to be within the GPU device hardware limitations: number of cores, threads queue depth, memory transfers, memory access conflicts, ... etc.

6. Increasing $N T$ enhances the solutions' qualities as it increases the Crossover and Mutation iterations. This could result in obtaining an optimal solution in less number of Evolve kernel calls, but with more time per kernel. For example, compare Tables 2 and 3.

7. We have experimented the Crossover and Mutation impacts independently from $N T$. In these experimentation, we determined the number of Crossover and Mutations iterations by an input parameter. We found that increasing the number of iterations lead to optimal solutions in less number of kernels, but with higher average kernel time. Tables 4 and 7 show the related results.

8. The Migration operation impact starts from the second Evolve kernel and onward. As per our algorithm design, the number of chromosomes to be migrated to a next population is proportional to $N B$. Our experimentation indicated that the number of chromosomes to be migrated from each block and their distribution over the next kernel blocks influence the solution quality obtained by that kernel. We experienced these impacts while testing the Pmed, Chess Board, and Large Duality Gap-C benchmarks 
as they required more kernel calls than the other benchmarks, refer to Tables 1,3 , and 8 .

9. The experimentation results are consistent with the time complexity analysis in Section 5 except for the results shown in Tables 4 and 7 as explained above. The Evolve kernel average time is proportional to $n, m$, and $T N$. Furthermore, the experimentation indicated that this average time is also proportional to $\frac{T B \times T N}{\text { Number of Cores }}$. This is valid because threads will be queued as the number of threads exceeds the number of available cores. Tables 2 and 5 show the impact of increasing $n$ and $m$ on the kernel time when fixing $N T$ and $N B$, while Tables 2 and 6 point out the impact of increasing $\frac{T B \times T N}{\text { Number of Cores }}$.

\section{Conclusions}

In this paper, we present a new genetic algorithm for the PMP based on GPU and pseudo-Boolean formulation. The algorithm is composed of Host code and Device code. The host randomly generates a population of chromosomes (candidate solutions) and passes them to a device kernel for fitness evaluations and enhancements. This basically iterates with migrating the best fit chromosomes from the current population to the next. The algorithm terminates at reaching an iteration limit or over saturating the solution enhancement. The algorithm is implemented using Cuda C version 7.5, and it was tested on 290 different benchmark instances. It has succeeded in obtaining optimal solutions for all the 290 instances except two for which better than $99.9 \%$ approximations have been obtained.

There are several venues for our future work on this topic. First, we will be working on identifying and developing solution enhancement operations that shall improve our algorithm performance. Second, we shall analyze and experiment the algorithm scalability limits on different GPGPU platforms. Third, we will investigate applying the presented algorithm on different variations of the facility location problems.

\section{References}

[1] Cuda-C Programming Guide, http: //docs.nvidia. com/cuda/cuda-c-programming-guide.

[2] Discrete location Problems Benchmark library, The P-median Problem, www.math.nsc.ru/AP/benchmarks/P-median.

[3] CURAND LIBRARY Programming Guide. NVIDIA, September 2015.

[4] Bader AlBdaiwi, Diptesh Ghosh, and Boris Glodengorin. Data aggregation for p-median problems. Journal of Combinatorial Optimization, 21:348$363,2011$. 


\begin{tabular}{|c|c|c|c|c|c|c|}
\hline $\begin{array}{l}\text { Instance } \\
\text { Code }\end{array}$ & $\mathrm{n}=\mathrm{m}$ & $p$ & $\begin{array}{c}\text { Number of } \\
\text { Potential Solutions }\end{array}$ & $\begin{array}{c}\text { Obtained Solution } \\
\text { Approximation Ratio }\end{array}$ & $\begin{array}{c}\text { Number of } \\
\text { Kernel Calls }\end{array}$ & $\begin{array}{l}\text { Time } \\
\text { (Sec.) }\end{array}$ \\
\hline Pmed 1 & 100 & 5 & $7.53 \mathrm{E}+07$ & Optimal & 1 & 2 \\
\hline Pmed 2 & 100 & 10 & $1.73 \mathrm{E}+13$ & Optimal & 1 & 2 \\
\hline Pmed 3 & 100 & 10 & $1.73 \mathrm{E}+13$ & Optimal & 1 & 2 \\
\hline Pmed 4 & 100 & 20 & $5.36 \mathrm{E}+20$ & Optimal & 1 & 2 \\
\hline Pmed 5 & 100 & 33 & $2.95 \mathrm{E}+26$ & Optimal & 2 & 4 \\
\hline Pmed 6 & 200 & 5 & $2.54 \mathrm{E}+09$ & Optimal & 1 & 8 \\
\hline Pmed 7 & 200 & 10 & $2.25 \mathrm{E}+16$ & Optimal & 1 & 6 \\
\hline Pmed 8 & 200 & 20 & $1.61 \mathrm{E}+27$ & Optimal & 4 & 15 \\
\hline Pmed 9 & 200 & 40 & $2.05 \mathrm{E}+42$ & Optimal & 7 & 23 \\
\hline Pmed 10 & 200 & 67 & $1.45 \mathrm{E}+54$ & Optimal & 13 & 49 \\
\hline Pmed 11 & 300 & 5 & $1.96 \mathrm{E}+10$ & Optimal & 1 & 13 \\
\hline Pmed 12 & 300 & 10 & $1.40 \mathrm{E}+18$ & Optimal & 2 & 19 \\
\hline Pmed 13 & 300 & 30 & $1.73 \mathrm{E}+41$ & Optimal & 10 & 61 \\
\hline Pmed 14 & 300 & 60 & $9.04 \mathrm{E}+63$ & Optimal & 14 & 137 \\
\hline Pmed 15 & 300 & 100 & $4.16 \mathrm{E}+81$ & Optimal & 16 & 3744 \\
\hline Pmed 16 & 400 & 5 & $8.32 \mathrm{E}+10$ & Optimal & 1 & 23 \\
\hline Pmed 17 & 400 & 10 & $2.58 \mathrm{E}+19$ & Optimal & 4 & 61 \\
\hline Pmed 18 & 400 & 40 & $1.97 \mathrm{E}+55$ & Optimal & 15 & 158 \\
\hline Pmed 19 & 400 & 80 & $4.23 \mathrm{E}+85$ & Optimal & 15 & 2608 \\
\hline Pmed 20 & 400 & 133 & $1.26 \mathrm{E}+109$ & Optimal & 17 & 462 \\
\hline Pmed 21 & 500 & 5 & $2.55 \mathrm{E}+11$ & Optimal & 1 & 34 \\
\hline Pmed 22 & 500 & 10 & $2.46 \mathrm{E}+20$ & Optimal & 6 & 150 \\
\hline Pmed 23 & 500 & 50 & $2.31 \mathrm{E}+69$ & Optimal & 27 & 495 \\
\hline Pmed 24 & 500 & 100 & $2.04 \mathrm{E}+107$ & Optimal & 16 & 4104 \\
\hline Pmed 25 & 500 & 167 & $7.85 \mathrm{E}+136$ & Optimal & 14 & 2201 \\
\hline Pmed 26 & 600 & 5 & $6.37 \mathrm{E}+11$ & Optimal & 1 & 50 \\
\hline Pmed 27 & 600 & 10 & $1.55 \mathrm{E}+21$ & Optimal & 9 & 280 \\
\hline Pmed 28 & 600 & 60 & $2.77 \mathrm{E}+83$ & Optimal & 13 & 2918 \\
\hline Pmed 29 & 600 & 120 & $1.01 \mathrm{E}+129$ & Optimal & 50 & 8856 \\
\hline Pmed 30 & 600 & 200 & $2.51 \mathrm{E}+164$ & 0.999497487 & 100 & 41687 \\
\hline Pmed 31 & 700 & 5 & $1.38 \mathrm{E}+12$ & Optimal & 1 & 98 \\
\hline Pmed 32 & 700 & 10 & $7.30 \mathrm{E}+21$ & Optimal & 3 & 186 \\
\hline Pmed 33 & 700 & 70 & $3.37 \mathrm{E}+97$ & Optimal & 28 & 5385 \\
\hline Pmed 34 & 700 & 140 & $5.03 \mathrm{E}+150$ & Optimal & 33 & 8143 \\
\hline Pmed 35 & 800 & 5 & $2.70 \mathrm{E}+12$ & Optimal & 2 & 249 \\
\hline Pmed 36 & 800 & 10 & $2.80 \mathrm{E}+22$ & Optimal & 2 & 163 \\
\hline Pmed 37 & 800 & 80 & $4.14 \mathrm{E}+111$ & Optimal & 15 & 8326 \\
\hline Pmed 38 & 900 & 5 & $4.87 \mathrm{E}+12$ & Optimal & 5 & 763 \\
\hline Pmed 39 & 900 & 10 & $9.14 \mathrm{E}+22$ & Optimal & 7 & 643 \\
\hline Pmed 40 & 900 & 90 & $5.13 \mathrm{E}+125$ & 0.99980503 & 100 & 63088 \\
\hline
\end{tabular}

Table 1: Results for $\mathrm{P}$ median benchmark instances obtained from OR-Library with $\mathrm{NB}=60$ and $\mathrm{NT}=256$. 


\begin{tabular}{|c|c|c|c|c|c|c|}
\hline $\begin{array}{c}\text { Instance } \\
\text { Code }\end{array}$ & $\mathrm{n}=\mathrm{m}$ & $\mathrm{p}$ & $\begin{array}{c}\text { Number of } \\
\text { Potential Solutions }\end{array}$ & $\begin{array}{c}\text { Obtained Solution } \\
\text { Approximation Ratio }\end{array}$ & $\begin{array}{c}\text { Number of } \\
\text { Kernel Calls }\end{array}$ & $\begin{array}{c}\text { Time } \\
\text { (Sec.) }\end{array}$ \\
\hline 313 & 128 & 16 & $9.3343 \mathrm{E}+19$ & Optimal & 1 & 7 \\
323 & 128 & 16 & $9.3343 \mathrm{E}+19$ & Optimal & 1 & 7 \\
334 & 128 & 16 & $9.3343 \mathrm{E}+19$ & Optimal & 1 & 7 \\
434 & 128 & 16 & $9.3343 \mathrm{E}+19$ & Optimal & 1 & 7 \\
534 & 128 & 16 & $9.3343 \mathrm{E}+19$ & Optimal & 1 & 7 \\
634 & 128 & 16 & $9.3343 \mathrm{E}+19$ & Optimal & 2 & 12 \\
734 & 128 & 16 & $9.3343 \mathrm{E}+19$ & Optimal & 1 & 7 \\
834 & 128 & 16 & $9.3343 \mathrm{E}+19$ & Optimal & 1 & 7 \\
934 & 128 & 16 & $9.3343 \mathrm{E}+19$ & Optimal & 1 & 7 \\
1034 & 128 & 16 & $9.3343 \mathrm{E}+19$ & Optimal & 1 & 7 \\
1134 & 128 & 16 & $9.3343 \mathrm{E}+19$ & Optimal & 1 & 7 \\
1234 & 128 & 16 & $9.3343 \mathrm{E}+19$ & Optimal & 1 & 6 \\
1334 & 128 & 16 & $9.3343 \mathrm{E}+19$ & Optimal & 1 & 7 \\
1434 & 128 & 16 & $9.3343 \mathrm{E}+19$ & Optimal & 1 & 7 \\
1534 & 128 & 16 & $9.3343 \mathrm{E}+19$ & Optimal & 1 & 7 \\
1634 & 128 & 16 & $9.3343 \mathrm{E}+19$ & Optimal & 1 & 6 \\
1734 & 128 & 16 & $9.3343 \mathrm{E}+19$ & Optimal & 2 & 13 \\
1834 & 128 & 16 & $9.3343 \mathrm{E}+19$ & Optimal & 1 & 7 \\
1934 & 128 & 16 & $9.3343 \mathrm{E}+19$ & Optimal & 1 & 7 \\
2034 & 128 & 16 & $9.3343 \mathrm{E}+19$ & Optimal & 1 & 7 \\
2134 & 128 & 16 & $9.3343 \mathrm{E}+19$ & Optimal & 1 & 7 \\
2234 & 128 & 16 & $9.3343 \mathrm{E}+19$ & Optimal & 1 & 7 \\
2334 & 128 & 16 & $9.3343 \mathrm{E}+19$ & Optimal & 1 & 7 \\
2434 & 128 & 16 & $9.3343 \mathrm{E}+19$ & Optimal & 1 & 7 \\
2534 & 128 & 16 & $9.3343 \mathrm{E}+19$ & Optimal & 1 & 7 \\
2634 & 128 & 16 & $9.3343 \mathrm{E}+19$ & Optimal & 1 & 7 \\
2734 & 128 & 16 & $9.3343 \mathrm{E}+19$ & Optimal & 1 & 7 \\
2834 & 128 & 16 & $9.3343 \mathrm{E}+19$ & Optimal & 1 & 7 \\
2934 & 128 & 16 & $9.3343 \mathrm{E}+19$ & Optimal & 1 & 7 \\
3034 & 128 & 16 & $9.3343 \mathrm{E}+19$ & Optimal & 1 & 7 \\
\hline
\end{tabular}

Table 2: Results for Perfect Codes Instances obtained from Discrete Location Problems Benchmark Library with NB $=120$ and NT $=256$. 


\begin{tabular}{|c|c|c|c|c|c|c|}
\hline $\begin{array}{c}\text { Instance } \\
\text { Code }\end{array}$ & $\mathrm{n}=\mathrm{m}$ & $\mathrm{p}$ & $\begin{array}{c}\text { Number of } \\
\text { Potential Solutions }\end{array}$ & $\begin{array}{c}\text { Obtained Solution } \\
\text { Approximation Ratio }\end{array}$ & $\begin{array}{l}\text { Number of } \\
\text { Kernel Calls }\end{array}$ & $\begin{array}{l}\text { Time } \\
\text { (Sec.) }\end{array}$ \\
\hline 334 & 144 & 16 & $6.88 \mathrm{E}+20$ & Optimal & 11 & 36 \\
\hline 434 & 144 & 16 & $6.88 \mathrm{E}+20$ & Optimal & 12 & 39 \\
\hline 534 & 144 & 16 & $6.88 \mathrm{E}+20$ & Optimal & 11 & 59 \\
\hline 634 & 144 & 16 & $6.88 \mathrm{E}+20$ & Optimal & 14 & 45 \\
\hline 734 & 144 & 16 & $6.88 \mathrm{E}+20$ & Optimal & 7 & 25 \\
\hline 834 & 144 & 16 & $6.88 \mathrm{E}+20$ & Optimal & 9 & 49 \\
\hline 934 & 144 & 16 & $6.88 \mathrm{E}+20$ & Optimal & 13 & 42 \\
\hline 1034 & 144 & 16 & $6.88 \mathrm{E}+20$ & Optimal & 29 & 100 \\
\hline 1134 & 144 & 16 & $6.88 \mathrm{E}+20$ & Optimal & 7 & 24 \\
\hline 1234 & 144 & 16 & $6.88 \mathrm{E}+20$ & Optimal & 21 & 67 \\
\hline 1334 & 144 & 16 & $6.88 \mathrm{E}+20$ & Optimal & 8 & 29 \\
\hline 1434 & 144 & 16 & $6.88 \mathrm{E}+20$ & Optimal & 10 & 34 \\
\hline 1534 & 144 & 16 & $6.88 \mathrm{E}+20$ & Optimal & 19 & 5 \\
\hline 1634 & 144 & 16 & $6.88 \mathrm{E}+20$ & Optimal & 18 & 5 \\
\hline 1734 & 144 & 16 & $6.88 \mathrm{E}+20$ & Optimal & 18 & 56 \\
\hline 1834 & 144 & 16 & $6.88 \mathrm{E}+20$ & Optimal & 46 & 142 \\
\hline 1934 & 144 & 16 & $6.88 \mathrm{E}+20$ & Optimal & 8 & 44 \\
\hline 2034 & 144 & 16 & $6.88 \mathrm{E}+20$ & Optimal & 11 & 37 \\
\hline 2134 & 144 & 16 & $6.88 \mathrm{E}+20$ & Optimal & 14 & 44 \\
\hline 2234 & 144 & 16 & $6.88 \mathrm{E}+20$ & Optimal & 22 & 75 \\
\hline 2334 & 144 & 16 & $6.88 \mathrm{E}+20$ & Optimal & 13 & 69 \\
\hline 2434 & 144 & 16 & $6.88 \mathrm{E}+20$ & Optimal & 7 & 23 \\
\hline 2534 & 144 & 16 & $6.88 \mathrm{E}+20$ & Optimal & 11 & 35 \\
\hline 2634 & 144 & 16 & $6.88 \mathrm{E}+20$ & Optimal & 2 & 9 \\
\hline 2734 & 144 & 16 & $6.88 \mathrm{E}+20$ & Optimal & 6 & 23 \\
\hline 2834 & 144 & 16 & $6.88 \mathrm{E}+20$ & Optimal & 6 & 20 \\
\hline 2934 & 144 & 16 & $6.88 \mathrm{E}+20$ & Optimal & 1 & 6 \\
\hline 3034 & 144 & 16 & $6.88 \mathrm{E}+20$ & Optimal & 17 & 54 \\
\hline 3134 & 144 & 16 & $6.88 \mathrm{E}+20$ & Optimal & 11 & 37 \\
\hline 3234 & 144 & 16 & $6.88 \mathrm{E}+20$ & Optimal & 10 & 33 \\
\hline
\end{tabular}

Table 3: Results for Chess Board Instances obtained from Discrete Location Problems Benchmark Library with NB $=480$ and NT $=96$. 


\begin{tabular}{|c|c|c|c|c|c|c|}
\hline $\begin{array}{c}\text { Instance } \\
\text { Code }\end{array}$ & $\mathrm{n}=\mathrm{m}$ & $\mathrm{p}$ & $\begin{array}{c}\text { Number of } \\
\text { Potential Solutions }\end{array}$ & $\begin{array}{c}\text { Obtained Solution } \\
\text { Approximation Ratio }\end{array}$ & $\begin{array}{c}\text { Number of } \\
\text { Kernel Calls }\end{array}$ & $\begin{array}{c}\text { Time } \\
\text { (Sec.) }\end{array}$ \\
\hline 1 & 133 & 12 & $3.84 \mathrm{E}+16$ & Optimal & 1 & 78 \\
2 & 133 & 12 & $3.84 \mathrm{E}+16$ & Optimal & 1 & 78 \\
3 & 133 & 12 & $3.84 \mathrm{E}+16$ & Optimal & 1 & 81 \\
4 & 133 & 12 & $3.84 \mathrm{E}+16$ & Optimal & 1 & 78 \\
5 & 133 & 12 & $3.84 \mathrm{E}+16$ & Optimal & 1 & 76 \\
6 & 133 & 12 & $3.84 \mathrm{E}+16$ & Optimal & 1 & 79 \\
7 & 133 & 12 & $3.84 \mathrm{E}+16$ & Optimal & 1 & 75 \\
8 & 133 & 12 & $3.84 \mathrm{E}+16$ & Optimal & 1 & 76 \\
9 & 133 & 12 & $3.84 \mathrm{E}+16$ & Optimal & 1 & 76 \\
10 & 133 & 12 & $3.84 \mathrm{E}+16$ & Optimal & 1 & 78 \\
11 & 133 & 12 & $3.84 \mathrm{E}+16$ & Optimal & 1 & 75 \\
12 & 133 & 12 & $3.84 \mathrm{E}+16$ & Optimal & 1 & 76 \\
13 & 133 & 12 & $3.84 \mathrm{E}+16$ & Optimal & 1 & 76 \\
14 & 133 & 12 & $3.84 \mathrm{E}+16$ & Optimal & 1 & 81 \\
15 & 133 & 12 & $3.84 \mathrm{E}+16$ & Optimal & 1 & 76 \\
16 & 133 & 12 & $3.84 \mathrm{E}+16$ & Optimal & 1 & 77 \\
17 & 133 & 12 & $3.84 \mathrm{E}+16$ & Optimal & 1 & 75 \\
18 & 133 & 12 & $3.84 \mathrm{E}+16$ & Optimal & 1 & 80 \\
19 & 133 & 12 & $3.84 \mathrm{E}+16$ & Optimal & 1 & 75 \\
20 & 133 & 12 & $3.84 \mathrm{E}+16$ & Optimal & 1 & 77 \\
21 & 133 & 12 & $3.84 \mathrm{E}+16$ & Optimal & 1 & 77 \\
22 & 133 & 12 & $3.84 \mathrm{E}+16$ & Optimal & 1 & 75 \\
23 & 133 & 12 & $3.84 \mathrm{E}+16$ & Optimal & 1 & 77 \\
24 & 133 & 12 & $3.84 \mathrm{E}+16$ & Optimal & 1 & 74 \\
25 & 133 & 12 & $3.84 \mathrm{E}+16$ & Optimal & 1 & 78 \\
26 & 133 & 12 & $3.84 \mathrm{E}+16$ & Optimal & 1 & 74 \\
27 & 133 & 12 & $3.84 \mathrm{E}+16$ & Optimal & 1 & 78 \\
28 & 133 & 12 & $3.84 \mathrm{E}+16$ & Optimal & 1 & 79 \\
29 & 133 & 12 & $3.84 \mathrm{E}+16$ & Optimal & 1 & 73 \\
30 & 133 & 12 & $3.84 \mathrm{E}+16$ & Optimal & 1 & 76 \\
\hline
\end{tabular}

Table 4: Results for Finite Projective Planes Instances, $\mathrm{K}=11$ obtained from Discrete Location Problems Benchmark Library with NB $=120$ and NT $=256$. In this experiment, the number of crossover and mutation iterations were preset to 60 . 


\begin{tabular}{|c|c|c|c|c|c|c|}
\hline $\begin{array}{c}\text { Instance } \\
\text { Code }\end{array}$ & $\mathrm{n}=\mathrm{m}$ & $\mathrm{p}$ & $\begin{array}{c}\text { Number of } \\
\text { Potential Solutions }\end{array}$ & $\begin{array}{c}\text { Obtained Solution } \\
\text { Approximation Ratio }\end{array}$ & $\begin{array}{c}\text { Number of } \\
\text { Kernel Calls }\end{array}$ & $\begin{array}{c}\text { Time } \\
\text { (Sec.) }\end{array}$ \\
\hline 1 & 307 & 18 & $5.51 \mathrm{E}+28$ & Optimal & 1 & 39 \\
2 & 307 & 18 & $5.51 \mathrm{E}+28$ & Optimal & 1 & 40 \\
3 & 307 & 18 & $5.51 \mathrm{E}+28$ & Optimal & 1 & 40 \\
4 & 307 & 18 & $5.51 \mathrm{E}+28$ & Optimal & 1 & 40 \\
5 & 307 & 18 & $5.51 \mathrm{E}+28$ & Optimal & 1 & 40 \\
6 & 307 & 18 & $5.51 \mathrm{E}+28$ & Optimal & 6 & 212 \\
7 & 307 & 18 & $5.51 \mathrm{E}+28$ & Optimal & 1 & 39 \\
8 & 307 & 18 & $5.51 \mathrm{E}+28$ & Optimal & 1 & 39 \\
9 & 307 & 18 & $5.51 \mathrm{E}+28$ & Optimal & 1 & 39 \\
10 & 307 & 18 & $5.51 \mathrm{E}+28$ & Optimal & 1 & 39 \\
11 & 307 & 18 & $5.51 \mathrm{E}+28$ & Optimal & 1 & 38 \\
12 & 307 & 18 & $5.51 \mathrm{E}+28$ & Optimal & 2 & 73 \\
13 & 307 & 18 & $5.51 \mathrm{E}+28$ & Optimal & 2 & 73 \\
14 & 307 & 18 & $5.51 \mathrm{E}+28$ & Optimal & 1 & 39 \\
15 & 307 & 18 & $5.51 \mathrm{E}+28$ & Optimal & 2 & 74 \\
16 & 307 & 18 & $5.51 \mathrm{E}+28$ & Optimal & 2 & 71 \\
17 & 307 & 18 & $5.51 \mathrm{E}+28$ & Optimal & 1 & 39 \\
18 & 307 & 18 & $5.51 \mathrm{E}+28$ & Optimal & 1 & 39 \\
19 & 307 & 18 & $5.51 \mathrm{E}+28$ & Optimal & 1 & 39 \\
20 & 307 & 18 & $5.51 \mathrm{E}+28$ & Optimal & 1 & 40 \\
21 & 307 & 18 & $5.51 \mathrm{E}+28$ & Optimal & 1 & 39 \\
22 & 307 & 18 & $5.51 \mathrm{E}+28$ & Optimal & 5 & 178 \\
23 & 307 & 18 & $5.51 \mathrm{E}+28$ & Optimal & 4 & 140 \\
24 & 307 & 18 & $5.51 \mathrm{E}+28$ & Optimal & 1 & 40 \\
25 & 307 & 18 & $5.51 \mathrm{E}+28$ & Optimal & 1 & 39 \\
26 & 307 & 18 & $5.51 \mathrm{E}+28$ & Optimal & 1 & 38 \\
27 & 307 & 18 & $5.51 \mathrm{E}+28$ & Optimal & 2 & 75 \\
28 & 307 & 18 & $5.51 \mathrm{E}+28$ & Optimal & 1 & 40 \\
29 & 307 & 18 & $5.51 \mathrm{E}+28$ & Optimal & 1 & 41 \\
30 & 307 & 18 & $5.51 \mathrm{E}+28$ & Optimal & 1 & 39 \\
\hline
\end{tabular}

Table 5: Results for Finite Projective Planes Instances, $\mathrm{K}=17$ obtained from Discrete Location Problems Benchmark Library with NB =120 and NT = 256 . 


\begin{tabular}{|c|c|c|c|c|c|c|}
\hline $\begin{array}{c}\text { Instance } \\
\text { Code }\end{array}$ & $\mathrm{n}=\mathrm{m}$ & $\mathrm{p}$ & $\begin{array}{c}\text { Number of } \\
\text { Potential Solutions }\end{array}$ & $\begin{array}{c}\text { Obtained Solution } \\
\text { Approximation Ratio }\end{array}$ & $\begin{array}{c}\text { Number of } \\
\text { Kernel Calls }\end{array}$ & $\begin{array}{c}\text { Time } \\
\text { (Sec.) }\end{array}$ \\
\hline 332 & 100 & 12 & $1.05 \mathrm{E}+15$ & Optimal & 1 & 46 \\
432 & 100 & 12 & $1.05 \mathrm{E}+15$ & Optimal & 2 & 85 \\
532 & 100 & 12 & $1.05 \mathrm{E}+15$ & Optimal & 2 & 86 \\
632 & 100 & 12 & $1.05 \mathrm{E}+15$ & Optimal & 1 & 46 \\
732 & 100 & 12 & $1.05 \mathrm{E}+15$ & Optimal & 1 & 46 \\
832 & 100 & 12 & $1.05 \mathrm{E}+15$ & Optimal & 1 & 45 \\
932 & 100 & 12 & $1.05 \mathrm{E}+15$ & Optimal & 1 & 45 \\
1032 & 100 & 12 & $1.05 \mathrm{E}+15$ & Optimal & 1 & 60 \\
1132 & 100 & 12 & $1.05 \mathrm{E}+15$ & Optimal & 1 & 60 \\
1232 & 100 & 12 & $1.05 \mathrm{E}+15$ & Optimal & 1 & 52 \\
1332 & 100 & 12 & $1.05 \mathrm{E}+15$ & Optimal & 1 & 46 \\
1432 & 100 & 12 & $1.05 \mathrm{E}+15$ & Optimal & 1 & 47 \\
1532 & 100 & 12 & $1.05 \mathrm{E}+15$ & Optimal & 2 & 91 \\
1632 & 100 & 12 & $1.05 \mathrm{E}+15$ & Optimal & 1 & 48 \\
1732 & 100 & 12 & $1.05 \mathrm{E}+15$ & Optimal & 1 & 49 \\
1832 & 100 & 12 & $1.05 \mathrm{E}+15$ & Optimal & 7 & 304 \\
1932 & 100 & 12 & $1.05 \mathrm{E}+15$ & Optimal & 1 & 48 \\
2032 & 100 & 12 & $1.05 \mathrm{E}+15$ & Optimal & 1 & 48 \\
2132 & 100 & 12 & $1.05 \mathrm{E}+15$ & Optimal & 1 & 49 \\
2232 & 100 & 12 & $1.05 \mathrm{E}+15$ & Optimal & 1 & 49 \\
2332 & 100 & 12 & $1.05 \mathrm{E}+15$ & Optimal & 1 & 49 \\
2432 & 100 & 12 & $1.05 \mathrm{E}+15$ & Optimal & 1 & 49 \\
2532 & 100 & 12 & $1.05 \mathrm{E}+15$ & Optimal & 1 & 49 \\
2632 & 100 & 12 & $1.05 \mathrm{E}+15$ & Optimal & 2 & 91 \\
2732 & 100 & 12 & $1.05 \mathrm{E}+15$ & Optimal & 25 & 1027 \\
2832 & 100 & 12 & $1.05 \mathrm{E}+15$ & Optimal & 1 & 49 \\
2932 & 100 & 12 & $1.05 \mathrm{E}+15$ & Optimal & 1 & 45 \\
3032 & 100 & 12 & $1.05 \mathrm{E}+15$ & Optimal & 1 & 45 \\
3132 & 100 & 12 & $1.05 \mathrm{E}+15$ & Optimal & 1 & 43 \\
3232 & 100 & 12 & $1.05 \mathrm{E}+15$ & Optimal & 2 & 88 \\
\hline
\end{tabular}

Table 6: Results for Large Duality Gap-A Instances obtained from Discrete Location Problems Benchmark Library with NB $=1500$ and NT $=64$. 


\begin{tabular}{|c|c|c|c|c|c|c|}
\hline $\begin{array}{c}\text { Instance } \\
\text { Code }\end{array}$ & $\mathrm{n}=\mathrm{m}$ & $\mathrm{p}$ & $\begin{array}{c}\text { Number of } \\
\text { Potential Solutions }\end{array}$ & $\begin{array}{c}\text { Obtained Solution } \\
\text { Approximation Ratio }\end{array}$ & $\begin{array}{c}\text { Number of } \\
\text { Kernel Calls }\end{array}$ & $\begin{array}{l}\text { Time } \\
(\text { Sec.) }\end{array}$ \\
\hline 331 & 100 & 14 & $4.42 \mathrm{E}+16$ & Optimal & 2 & 54 \\
\hline 431 & 100 & 14 & $4.42 \mathrm{E}+16$ & Optimal & 2 & 54 \\
\hline 531 & 100 & 14 & $4.42 \mathrm{E}+16$ & Optimal & 2 & 52 \\
\hline 631 & 100 & 14 & $4.42 \mathrm{E}+16$ & Optimal & 2 & 58 \\
\hline 731 & 100 & 14 & $4.42 \mathrm{E}+16$ & Optimal & 5 & 130 \\
\hline 831 & 100 & 14 & $4.42 \mathrm{E}+16$ & Optimal & 1 & 27 \\
\hline 931 & 100 & 14 & $4.42 \mathrm{E}+16$ & Optimal & 2 & 58 \\
\hline 1031 & 100 & 14 & $4.42 \mathrm{E}+16$ & Optimal & 1 & 30 \\
\hline 1131 & 100 & 14 & $4.42 \mathrm{E}+16$ & Optimal & 1 & 30 \\
\hline 1231 & 100 & 14 & $4.42 \mathrm{E}+16$ & Optimal & 6 & 156 \\
\hline 1331 & 100 & 14 & $4.42 \mathrm{E}+16$ & Optimal & 1 & 29 \\
\hline 1431 & 100 & 14 & $4.42 \mathrm{E}+16$ & Optimal & 5 & 138 \\
\hline 1531 & 100 & 14 & $4.42 \mathrm{E}+16$ & Optimal & 6 & 171 \\
\hline 1631 & 100 & 14 & $4.42 \mathrm{E}+16$ & Optimal & 2 & 52 \\
\hline 1731 & 100 & 14 & $4.42 \mathrm{E}+16$ & Optimal & 1 & 30 \\
\hline 1831 & 100 & 14 & $4.42 \mathrm{E}+16$ & Optimal & 2 & 52 \\
\hline 1931 & 100 & 14 & $4.42 \mathrm{E}+16$ & Optimal & 1 & 26 \\
\hline 2031 & 100 & 14 & $4.42 \mathrm{E}+16$ & Optimal & 1 & 27 \\
\hline 2131 & 100 & 14 & $4.42 \mathrm{E}+16$ & Optimal & 1 & 26 \\
\hline 2231 & 100 & 14 & $4.42 \mathrm{E}+16$ & Optimal & 2 & 62 \\
\hline 2331 & 100 & 14 & $4.42 \mathrm{E}+16$ & Optimal & 1 & 28 \\
\hline 2431 & 100 & 14 & $4.42 \mathrm{E}+16$ & Optimal & 5 & 132 \\
\hline 2531 & 100 & 14 & $4.42 \mathrm{E}+16$ & Optimal & 1 & 27 \\
\hline 2631 & 100 & 14 & $4.42 \mathrm{E}+16$ & Optimal & 10 & 252 \\
\hline 2731 & 100 & 14 & $4.42 \mathrm{E}+16$ & Optimal & 2 & 61 \\
\hline 2831 & 100 & 14 & $4.42 \mathrm{E}+16$ & Optimal & 1 & 32 \\
\hline 2931 & 100 & 14 & $4.42 \mathrm{E}+16$ & Optimal & 15 & 387 \\
\hline 3031 & 100 & 14 & $4.42 \mathrm{E}+16$ & Optimal & 1 & 26 \\
\hline 3131 & 100 & 14 & $4.42 \mathrm{E}+16$ & Optimal & 1 & 30 \\
\hline 3231 & 100 & 14 & $4.42 \mathrm{E}+16$ & Optimal & 3 & 75 \\
\hline
\end{tabular}

Table 7: Results for Large Duality Gap-B Instances obtained from Discrete Location Problems Benchmark Library with $\mathrm{NB}=120$ and $\mathrm{NT}=256$. In this experiment, the number of crossover and mutation iterations was preset to 20 . 


\begin{tabular}{|c|c|c|c|c|c|c|}
\hline $\begin{array}{c}\text { Instance } \\
\text { Code }\end{array}$ & $\mathrm{n}=\mathrm{m}$ & $\mathrm{p}$ & $\begin{array}{c}\text { Number of } \\
\text { Potential Solutions }\end{array}$ & $\begin{array}{c}\text { Obtained Solution } \\
\text { Approximation Ratio }\end{array}$ & $\begin{array}{c}\text { Number of } \\
\text { Kernel Calls }\end{array}$ & $\begin{array}{c}\text { Time } \\
\text { (Sec.) }\end{array}$ \\
\hline 333 & 100 & 14 & $4.42 \mathrm{E}+16$ & Optimal & 3 & 37 \\
433 & 100 & 14 & $4.42 \mathrm{E}+16$ & Optimal & 2 & 26 \\
533 & 100 & 14 & $4.42 \mathrm{E}+16$ & Optimal & 2 & 26 \\
633 & 100 & 14 & $4.42 \mathrm{E}+16$ & Optimal & 4 & 47 \\
733 & 100 & 14 & $4.42 \mathrm{E}+16$ & Optimal & 3 & 38 \\
833 & 100 & 14 & $4.42 \mathrm{E}+16$ & Optimal & 3 & 37 \\
933 & 100 & 14 & $4.42 \mathrm{E}+16$ & Optimal & 2 & 26 \\
1033 & 100 & 14 & $4.42 \mathrm{E}+16$ & Optimal & 4 & 49 \\
1133 & 100 & 14 & $4.42 \mathrm{E}+16$ & Optimal & 7 & 80 \\
1233 & 100 & 14 & $4.42 \mathrm{E}+16$ & Optimal & 11 & 126 \\
1333 & 100 & 14 & $4.42 \mathrm{E}+16$ & Optimal & 20 & 218 \\
1433 & 100 & 14 & $4.42 \mathrm{E}+16$ & Optimal & 2 & 27 \\
1533 & 100 & 14 & $4.42 \mathrm{E}+16$ & Optimal & 32 & 363 \\
1633 & 100 & 14 & $4.42 \mathrm{E}+16$ & Optimal & 3 & 37 \\
1733 & 100 & 14 & $4.42 \mathrm{E}+16$ & Optimal & 2 & 26 \\
1833 & 100 & 14 & $4.42 \mathrm{E}+16$ & Optimal & 18 & 198 \\
1933 & 100 & 14 & $4.42 \mathrm{E}+16$ & Optimal & 3 & 36 \\
2033 & 100 & 14 & $4.42 \mathrm{E}+16$ & Optimal & 5 & 59 \\
2133 & 100 & 14 & $4.42 \mathrm{E}+16$ & Optimal & 7 & 81 \\
2233 & 100 & 14 & $4.42 \mathrm{E}+16$ & Optimal & 2 & 26 \\
2333 & 100 & 14 & $4.42 \mathrm{E}+16$ & Optimal & 4 & 47 \\
2433 & 100 & 14 & $4.42 \mathrm{E}+16$ & Optimal & 9 & 105 \\
2533 & 100 & 14 & $4.42 \mathrm{E}+16$ & Optimal & 22 & 245 \\
2633 & 100 & 14 & $4.42 \mathrm{E}+16$ & Optimal & 3 & 37 \\
2733 & 100 & 14 & $4.42 \mathrm{E}+16$ & Optimal & 78 & 873 \\
2833 & 100 & 14 & $4.42 \mathrm{E}+16$ & Optimal & 2 & 27 \\
2933 & 100 & 14 & $4.42 \mathrm{E}+16$ & Optimal & 8 & 92 \\
3033 & 100 & 14 & $4.42 \mathrm{E}+16$ & Optimal & 1 & 15 \\
3133 & 100 & 14 & $4.42 \mathrm{E}+16$ & Optimal & 2 & 26 \\
3233 & 100 & 14 & $4.42 \mathrm{E}+16$ & Optimal & 3 & 37 \\
\hline
\end{tabular}

Table 8: Results for Large Duality Gap-C Instances obtained from Discrete Location Problems Benchmark Library with NB $=1500$ and NT $=32$. 


\begin{tabular}{|c|c|c|c|c|c|}
\hline $\mathrm{n}=\mathrm{m}$ & $\mathrm{p}$ & $\begin{array}{c}\text { Number of } \\
\text { Potential Solutions }\end{array}$ & $\begin{array}{c}\text { Obtained Solution } \\
\text { Approximation Ratio }\end{array}$ & $\begin{array}{l}\text { Number of } \\
\text { Kernel Calls }\end{array}$ & $\begin{array}{l}\text { Time } \\
\text { (Sec.) }\end{array}$ \\
\hline 100 & 5 & $7.53 \mathrm{E}+07$ & Optimal & 1 & 10 \\
\hline 100 & 10 & $1.73 \mathrm{E}+13$ & Optimal & 1 & 11 \\
\hline 100 & 20 & $5.36 \mathrm{E}+20$ & Optimal & 1 & 11 \\
\hline 100 & 33 & $2.95 \mathrm{E}+26$ & Optimal & 1 & 9 \\
\hline 200 & 5 & $2.54 \mathrm{E}+09$ & Optimal & 1 & 41 \\
\hline 200 & 10 & $2.25 \mathrm{E}+16$ & Optimal & 1 & 39 \\
\hline 200 & 20 & $1.61 \mathrm{E}+27$ & Optimal & 1 & 40 \\
\hline 200 & 40 & $2.05 \mathrm{E}+42$ & Optimal & 1 & 62 \\
\hline 200 & 67 & $1.45 \mathrm{E}+54$ & Optimal & 1 & 50 \\
\hline 300 & 5 & $1.96 \mathrm{E}+10$ & Optimal & 1 & 86 \\
\hline 300 & 10 & $1.40 \mathrm{E}+18$ & Optimal & 1 & 143 \\
\hline 300 & 30 & $1.73 \mathrm{E}+41$ & Optimal & 1 & 83 \\
\hline 300 & 60 & $9.04 \mathrm{E}+63$ & Optimal & 1 & 95 \\
\hline 300 & 100 & $4.16 \mathrm{E}+81$ & Optimal & 1 & 111 \\
\hline 400 & 5 & $8.32 \mathrm{E}+10$ & Optimal & 1 & 256 \\
\hline 400 & 10 & $2.58 \mathrm{E}+19$ & Optimal & 1 & 253 \\
\hline 400 & 40 & $1.97 \mathrm{E}+55$ & Optimal & 1 & 147 \\
\hline 400 & 80 & $4.23 \mathrm{E}+85$ & Optimal & 1 & 172 \\
\hline 400 & 133 & $1.26 \mathrm{E}+109$ & Optimal & 1 & 197 \\
\hline 500 & 5 & $2.55 \mathrm{E}+11$ & Optimal & 1 & 401 \\
\hline 500 & 10 & $2.46 \mathrm{E}+20$ & Optimal & 1 & 389 \\
\hline 500 & 50 & $2.31 \mathrm{E}+69$ & Optimal & 2 & 430 \\
\hline 500 & 100 & $2.04 \mathrm{E}+107$ & Optimal & 2 & 486 \\
\hline 500 & 167 & $7.85 \mathrm{E}+136$ & Optimal & 1 & 313 \\
\hline 600 & 5 & $6.37 \mathrm{E}+11$ & Optimal & 1 & 579 \\
\hline 600 & 10 & $1.55 \mathrm{E}+21$ & Optimal & 2 & 604 \\
\hline 600 & 60 & $2.77 \mathrm{E}+83$ & Optimal & 2 & 617 \\
\hline 600 & 120 & $1.01 \mathrm{E}+129$ & Optimal & 2 & 706 \\
\hline 600 & 200 & $2.51 \mathrm{E}+164$ & Optimal & 1 & 453 \\
\hline 700 & 5 & $1.38 \mathrm{E}+12$ & Optimal & 2 & 846 \\
\hline 700 & 10 & $7.30 \mathrm{E}+21$ & Optimal & 2 & 818 \\
\hline 700 & 70 & $3.37 \mathrm{E}+97$ & Optimal & 2 & 839 \\
\hline 700 & 140 & $5.03 \mathrm{E}+150$ & Optimal & 2 & 960 \\
\hline 800 & 5 & $2.70 \mathrm{E}+12$ & Optimal & 3 & 1660 \\
\hline 800 & 10 & $2.80 \mathrm{E}+22$ & Optimal & 2 & 1507 \\
\hline 800 & 80 & $4.14 \mathrm{E}+111$ & Optimal & 2 & 1123 \\
\hline 900 & 5 & $4.87 \mathrm{E}+12$ & Optimal & 1 & 1291 \\
\hline 900 & 10 & $9.14 \mathrm{E}+22$ & Optimal & 2 & 1905 \\
\hline 900 & 90 & $5.13 \mathrm{E}+125$ & Optimal & 2 & 1407 \\
\hline
\end{tabular}

Table 9: Results for the Complex Instances Introduced in [16, where NB $=120$ and $\mathrm{NT}=256$. 
[5] Bader F AlBdaiwi, Boris Goldengorin, and Gerard Sierksma. Equivalent instances of the simple plant location problem. Computers $\&$ Mathematics with Applications, 57(5):812-820, 2009.

[6] Osman Alp and Erhan Erkut. An efficient genetic algorithm for the pmedian problem. Annals of Oerations Research, 122:21-42, 2003.

[7] Fabiano Fernandes Bargos, Wendell de Queiroz Lamas, Danubia Caporusso Bargos, Morun Bernardino Neto, and Paula Cristiane Pinto Mesquita Pardal. Location problem method applied to sugar and ethanol mills location optimization. Renewable and Sustainable Energy Reviews, 65:274-282, 2016.

[8] J. E. Beasley. OR-LIBRARY,http://people. brunel. ac.uk/ 〜mastjjb/jeb/orlib/pmedinfo. html

[9] Benjamin Biesinger, Bin Hu, and Günther Raidl. A hybrid genetic algorithm with solution archive for the discrete $(\mathrm{r} \mid \mathrm{p})$-centroid problem. Journal of Heuristics, 21(3):391-431, 2015.

[10] Burcin Bozkaya, Jianjun Zhang, and Erhan Erkut. An efficient genetic algorithm for the p-median problem. Facility location: Applications and theory, pages 179-205, 2002.

[11] Mark S. Daskin and Kayse Lee Maass. Location Science, G. Laporte, S. Nickel and F. Saldanha da Gama (Eds.), chapter 2: The p-Median Problem, pages 21-45. Springer, 2015.

[12] Zvi Drezner, Jack Brimberg, Nenad Mladenović, and Said Salhi. New heuristic algorithms for solving the planar p-median problem. Computers \& Operations Research, 62:296-304, 2015.

[13] Tarek A El-Mihoub, Adrian A Hopgood, Lars Nolle, and Alan Battersby. Hybrid genetic algorithms: A review. Engineering Letters, 13(2):124-137, 2006.

[14] Reza Zanjirani Farahani, Masoud Hekmatfar, Alireza Boloori Arabani, and Ehsan Nikbakhsh. Hub location problems: A review of models, classification, solution techniques, and applications. Computers \& Industrial Engineering, 64(4):1096-1109, 2013.

[15] Boris Goldengorin, Anton Kocheturov, and Panos M Pardalos. A pseudoboolean approach to the market graph analysis by means of the p-median model. In Clusters, Orders, and Trees: Methods and Applications, pages 77-89. Springer, 2014.

[16] Boris Goldengorin, Dmitry Krushinsky, and Panos Pardalos. Cell Formation in Industrial Engineering, Theory, Algorithms and Experiments. Springer, 2013. 
[17] Marshall Hall and Donald E Knuth. Combinatorial analysis and computers. The American Mathematical Monthly, 72(2):21-28, 1965.

[18] Peter Ladislaw Hammer. Plant location - a pseudo-boolean approach. Israel Journal of Technology, 6(5):330-332, 1968.

[19] HP Inc., USA. HP Z820 Workstation, April 2015. c04111526 - DA - 14264 - Worldwide - Version 48.

[20] Patrick Jaillet, Gao Song, and Gang Yu. Airline network design and hub location problems. Location science, 4(3):195-212, 1996.

[21] Jorge H Jaramillo, Joy Bhadury, and Rajan Batta. On the use of genetic algorithms to solve location problems. Computers $\&$ Operations Research, 29(6):761-779, 2002.

[22] Semin Kang, Sung-Soo Kim, Jongho Won, and Young-Min Kang. Gpubased parallel genetic approach to large-scale travelling salesman problem. Journal of Super Computing, pages 1-16, May 2016. DOI: 10.1007/s11227016-1748-1.

[23] Oded Kariv and S Louis Hakimi. An algorithmic approach to network location problems. II: The p-medians. SIAM Journal on Applied Mathematics, 37(3):539-560, 1979.

[24] Lev Aleksandrovich Kazakovtsev, Victor Orlov, Aljona Aleksandrovna Stupina, and Vladimir Kazakovtsev. Modied genetic algorithm with greedy heuristic for continuous and discrete p-median problems. Facta Universitatis, Series: Mathematics and Informatics, 30(1):89-106, 2015.

[25] Gilbert Laporte, Stefan Nickel, and Francisco Saldanha da Gama. Location science. Springer, 2015.

[26] Gino Lim and Likang Ma. Gpu-based parallel vertex substitution algorithm for the p-median problem. Computers 83 Industrial Engineering, 64(1):381388, January 2013.

[27] Likang Ma and Gino Lim. Gpu-based parallel computational algorithms for solving p-median problem. In IIE Annual Conference. Proceedings, page 1. Institute of Industrial Engineers-Publisher, 2011.

[28] James McCaffrey. Generating the mth lexicographical element of a mathematical combination. CTAN: http://msdn. microsoft. com/en-us/library/aa289166, July 2004.

[29] Melanie Mitchell. An Introduction to Genetic Algorithms. MIT Press, Cambridge, 1998.

[30] Nenad Mladenović, Jack Brimberg, Pierre Hansen, and José A MorenoPérez. The p-median problem: A survey of metaheuristic approaches. European Journal of Operational Research, 179(3):927-939, 2007. 
[31] Nvidia Corporation, USA. Tesla K40 Active Accelerator, November 2013. BD-06949-001_v03.

[32] Pascal Rebreyend, Laurent Lemarchand, and Reinhardt Euler. A computational comparison of different algorithms for very large p-median problems. In $15^{\text {th }}$ European Conference on Evolutionary Computation in Combinatorial Optimization, pages 13-24. Springer, 2015.

[33] Josh Reese. Solution methods for the p-median problem: An annotated bibliography. Networks, 48(3):125-142, 2006.

[34] Yonglin Ren and Anjali Awasthi. Investigating metaheuristics applications for capacitated location allocation problem on logistics networks. In Chaos Modeling and Control Systems Design, pages 213-238. Springer, 2015.

[35] Mauricio GC Resende and Renato F Werneck. A hybrid heuristic for the p-median problem. Journal of heuristics, 10(1):59-88, 2004.

[36] Zorica Stanimirović. A genetic algorithm approach for the capacitated single allocation p-hub median problem. Computing and Informatics, 29(1):117-132, 2012.

[37] Raca Todosijević, Dragan Urošević, Nenad Mladenović, and Saïd Hanafi. A general variable neighborhood search for solving the uncapacitated rallocation p-hub median problem. Optimization Letters, pages 1-13, 2015. 